Article

\title{
A Y-Shaped Microfluidic Device to Study the Combined Effect of Wall Shear Stress and ATP Signals on Intracellular Calcium Dynamics in Vascular Endothelial Cells
}

\author{
Zong-Zheng Chen ${ }^{1}$, Zheng-Ming Gao ${ }^{1}$, De-Pei Zeng ${ }^{1}$, Bo Liu ${ }^{1}$, Yong Luan ${ }^{2, *}$ \\ and Kai-Rong Qin ${ }^{1, *}$ \\ 1 Department of Biomedical Engineering, Dalian University of Technology, Dalian 116024, China; \\ zongzheng@mail.dlut.edu.cn (Z.-Z.C.); gaozhengming@mail.dlut.edu.cn (Z.-M.G.); \\ 1925044995@mail.dlut.edu.cn (D.-P.Z.); lbo@dlut.edu.cn (B.L.) \\ 2 Department of Anesthesiology, The First Affiliated Hospital of Dalian Medical University, \\ Dalian 116011, China \\ * Correspondence: cclyyly@hotmail.com (Y.L.); krqin@dlut.edu.cn (K.-R.Q.); \\ Tel.: +86-8363-5963-2054 (Y.L.); +86-411-8470-9690 (K.-R.Q.)
}

Academic Editors: Weihua Li, Hengdong Xi and Say Hwa Tan Received: 13 October 2016; Accepted: 18 November 2016; Published: 23 November 2016

\begin{abstract}
The intracellular calcium dynamics in vascular endothelial cells (VECs) in response to wall shear stress (WSS) and/or adenosine triphosphate (ATP) have been commonly regarded as an important factor in regulating VEC function and behavior including proliferation, migration and apoptosis. However, the effects of time-varying ATP signals have been usually neglected in the past investigations in the field of VEC mechanobiology. In order to investigate the combined effects of WSS and dynamic ATP signals on the intracellular calcium dynamic in VECs, a Y-shaped microfluidic device, which can provide the cultured cells on the bottom of its mixing micro-channel with stimuli of WSS signal alone and different combinations of WSS and ATP signals in one single micro-channel, is proposed. Both numerical simulation and experimental studies verify the feasibility of its application. Cellular experimental results also suggest that a combination of WSS and ATP signals rather than a WSS signal alone might play a more significant role in $\mathrm{VEC} \mathrm{Ca}^{2+}$ signal transduction induced by blood flow.
\end{abstract}

Keywords: Y-shaped microfluidic device; wall shear stress; adenosine triphosphate (ATP) signal; combined effect; vascular endothelial cells; calcium dynamics

\section{Introduction}

Vascular endothelial cells (VECs) lining the innermost layer of vessel walls directly contact the flowing blood and thus are exposed to both wall shear stress (WSS) induced by blood flow and adenosine triphosphate (ATP) contained in the blood. A number of investigations have revealed that this shear stress, either alone or along with the presence of ATP, activates the dynamic response of intracellular calcium ion $\left(\mathrm{Ca}^{2+}\right)$ signaling system in VECs [1-10]. From systemic dynamic point of view, the VEC intracellular $\mathrm{Ca}^{2+}$ signaling system can be considered as a dynamic system, with the WSS and ATP stimuli as the input signals and the intracellular $\mathrm{Ca}^{2+}$ dynamic response as the output signal of the dynamic system [11]. The intracellular $\mathrm{Ca}^{2+}$ dynamics in VECs motivated by WSS and/or ATP have been commonly regarded as a critical factor in regulating VEC function and behavior including proliferation, migration and apoptosis [12-14]. Therefore, it is of significance to experimentally investigate the VEC intracellular $\mathrm{Ca}^{2+}$ dynamics induced by WSS and/or ATP from systemic dynamic point of view. 
In blood circulatory systems in vivo, the WSS and ATP signals are very complicated because they are influenced by many interference factors. In vitro experimental studies could exclude these interference factors existing in vivo. Since the 1980s, an in vitro flow shear device, namely parallel-plate flow chamber (PPFC), of which the height is far smaller than the width and the length, has been usually adopted to quantitatively simulate the WSS and ATP in the extracellular microenvironments and investigate the intracellular $\mathrm{Ca}^{2+}$ dynamics in VECs in response to WSS and/or ATP [1-10]. Using the PPFC, it has been demonstrated that the dynamic behavior of the VEC intracellular $\mathrm{Ca}^{2+}$ in response to WSS could be modulated by extracellular ATP in a dose-dependent manner [1-9]. However, all these excellent investigations have focused on either WSS alone or WSS together with a constant ATP concentration. The effects of dynamic ATP signals, which would be pivotal in the VEC intracellular $\mathrm{Ca}^{2+}$ signaling system, have been totally neglected in these researches by PPFCs.

In recent years, with the development of micro- and nano-technology, microfluidics has been becoming an emerging bioengineering approach to study cellular dynamics with the ability of precisely controlling the spatial and/or temporal distribution of biochemical factors in one microfluidic channel [15-20]. Using a microfluidic device, Bibhas et al. characterized the spatiotemporal evolution of intracellular calcium "flickers" in response to steady, pulsatile, or oscillatory WSS through a frequency controlled solenoid valve [15]; however, the effects of the flowing media containing biochemical signals (e.g., ATP signal) were missing in their studies. A number of investigations by microfluidics have studied the effects of dynamic biochemical signals on the function and behavior of biological cells [16-20]. For instance, Yamada et al. invented a Y-shaped microfluidic device for rapidly switching ATP solution or no ATP solution on HEK293 cells, and studied the intracellular $\mathrm{Ca}^{2+}$ response following dynamic ATP signal [16]. However, all these studies have not fully considered the influence of WSS signal on the biological cells [17-20].

In order to efficiently investigate the combined effect of WSS and ATP signals on the VEC intracellular calcium dynamics, particularly the effect of dynamic ATP signals together with WSS signal, a Y-shaped microfluidic device, which possesses an inlet A with inflowing static or dynamic ATP signal and an inlet $B$ without ATP signal, is designed based upon the principles of fluid mechanics and mass transfer (Figure 1a). In this microfluidic device, the flow rate in the inlet $A$ is constant but that in the inlet B is time-varying. Therefore, different types of stimulating signals, including static or dynamic WSS alone, dynamic WSS signal together with static ATP signal, static WSS signal together with dynamic ATP signal, could be implemented in the mixing micro-channel C. The implementation of the different types of dynamic biochemical signals was experimentally validated by fluorescent signals which could be easily observed by a fluorescence microscope with a charge-coupled device (CCD) camera (DS126431, Canon Inc., Tokyo, Japan). Finally, the dynamic responses of the intracellular $\mathrm{Ca}^{2+}$ in human umbilical vein endothelial cells (HUVECs) in exposure to the different kinds of stimulating signals were detected using the proposed microfluidic device.

(a)

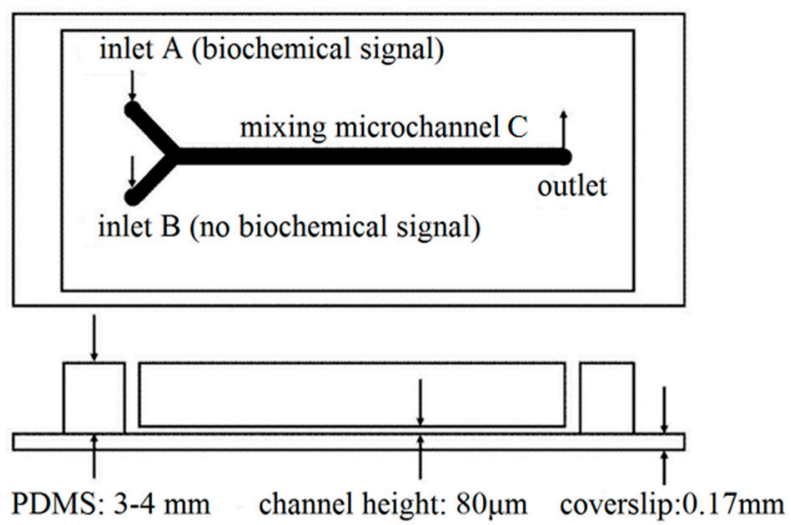

Figure 1. Cont. 
(b)

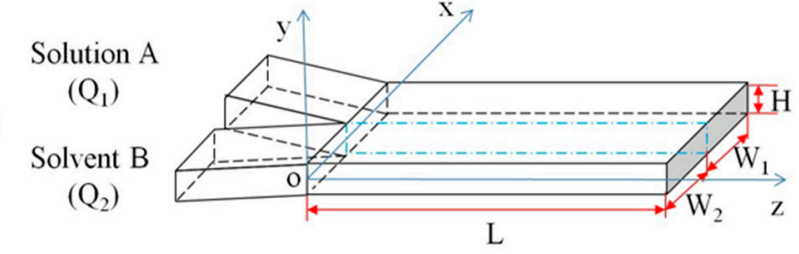

biochemical signal generator

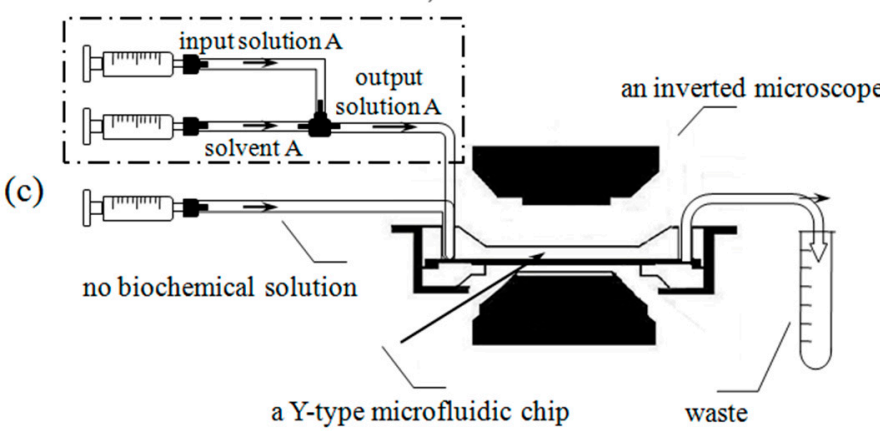

Figure 1. Schematic diagram of a Y-shaped microfluidic device. (a) Polydimethylsiloxane (PDMS)-glass structure; (b) coordinate system; (c) the integrated experimental system.

\section{Materials and Methods}

\subsection{Equations Governing Pulsatile Flow and Mass Transfer}

The geometry and the rectangular coordinate system oxyz of the shallow Y-shaped microfluidic chip used in this study are illustrated in Figure $1 \mathrm{~b}$. It is assumed that the height, $H$, is far smaller than the width, $W$, and the length, $L$, of the mixing micro-channel. A solution A with dynamic biochemical signal and a solution $B$ without biochemical signal were driven into the inlet $A$ and $B$ by two programmable syringe pumps, respectively.

\subsubsection{Equation Governing Pulsatile Flow in the Mixing Micro-Channel C}

It is assumed that both of the two solutions are Newtonian fluids with identical viscosity, the pulsatile flow in the mixing micro-channel $\mathrm{C}$ driven by the programmable syringe pumps is a fully developed laminar flow. By neglecting the boundary effects from the lateral sides and the ends, the equation governing the pulsatile flow in the mixing micro-channel can be simplified as,

$$
\frac{\partial u}{\partial t}=-\frac{1}{\rho} \frac{\partial p}{\partial z}+\frac{\eta}{\rho} \frac{\partial^{2} u}{\partial y^{2}}
$$

where, $u=u(y, t)$ is the fluid velocity along $z$-direction, $p=p(z, t)$ is the pressure, $t$ is the time, $\eta$ is the fluid viscosity, $\rho$ is the fluid density.

With the assumption of quasi-steady flow, the velocity profile $u(y, t)$, the height-wise averaging velocity $\bar{u}$ and the shear stress $\tau_{w}(t)$ can be given by [21],

$$
\begin{gathered}
u(y, t)=\frac{3}{2 W H}\left[1-\left(\frac{2 y}{H}\right)^{2}\right] Q(t) \\
\bar{u}(t)=\frac{Q(t)}{W H} \\
\tau_{w}(t)=\frac{6 \mu Q(t)}{W H^{2}}
\end{gathered}
$$

where $Q(t)$ is the total flow rate through the mixing micro-channel C. 


\subsubsection{Control of Two-Stream Flow Widths in the Mixing Micro-Channel C}

It is assumed that solution $\mathrm{A}$ has a constant volume flow rate $Q_{\mathrm{A}}$, and solvent $\mathrm{B}$ has a dynamically changing volume flow rate $Q_{\mathrm{B}}$. The flow velocity $u_{\mathrm{A}}$ of solution $\mathrm{A}$ is the same as the flow velocity $u_{\mathrm{B}}$ of solvent $B$, which satisfies [22],

$$
u_{\mathrm{A}}(y, t)=u_{\mathrm{B}}(y, t)=\frac{3}{2 W H}\left[1-\left(\frac{2 y}{H}\right)^{2}\right] Q(t)
$$

where $Q(t)=Q_{\mathrm{A}}+Q_{\mathrm{B}}(t)$, the volume flow rates $Q_{\mathrm{A}}$ and $Q_{\mathrm{B}}(t)$ satisfy [22],

$$
\frac{W_{1}}{W_{2}}=\frac{Q_{\mathrm{A}}}{Q_{\mathrm{B}}(t)}
$$

where $W_{1}$ and $W_{2}$ are the widths of the solution $A$ and solvent $B$, respectively, $W=W_{1}+W_{2}$. Equation (6) shows that the ratio of the widths $W_{1} / W_{2}$ of two streams in the mixing channel is uniquely determined by the externally controlled flow rate ratio $Q_{\mathrm{A}} / Q_{\mathrm{B}}(t)$. In this work, the flow rate ratio is set to be $Q_{\mathrm{A}} / Q_{\mathrm{B}}(t)=(1-\varepsilon(t)) / \varepsilon(t)$. Hence, the width of the solvent $\mathrm{B}$ in the Y-shaped channel is $\varepsilon W$, which primarily determines the inlet boundary $(z=0)$ of biochemical flow in the mixing micro-channel C.

\subsubsection{Taylor-Aris Dispersion in the Mixing Micro-Channel C}

In the mixing micro-channel $C$ where molecules are mixed by diffusion, the concentration $\phi$ of a biochemical substance is governed by [22],

$$
\frac{\partial \phi}{\partial t}+u(y, t) \frac{\partial \phi}{\partial z}=D\left(\frac{\partial^{2} \phi}{\partial x^{2}}+\frac{\partial^{2} \phi}{\partial y^{2}}+\frac{\partial^{2} \phi}{\partial z^{2}}\right)
$$

where $\phi=\phi(x, y, z, t)$ is the concentration of biochemical substance, $D$ is molecular diffusivity coefficient. Because the height of the micro-channel is smaller, a uniform concentration distribution of biochemical substance is easily formed in the $y$ direction. Therefore, in this study, we only consider the height-wise averaging concentration, $\bar{\phi}=\bar{\phi}(x, z, t)$, defined as

$$
\bar{\phi}(x, z, t)=\frac{1}{H} \int_{-H / 2}^{H / 2} \phi(x, y, z, t) \mathrm{d} y
$$

The transportation of the height-wise averaging concentration $\bar{\phi}$ in the mixing micro-channel $C$ is governed by the following Taylor-Aris dispersion equation [22],

$$
\frac{\partial \bar{\phi}}{\partial t}+\bar{u} \frac{\partial \bar{\phi}}{\partial z}=D \frac{\partial^{2} \bar{\phi}}{\partial x^{2}}+D_{\mathrm{eff}} \frac{\partial^{2} \bar{\phi}}{\partial z^{2}}
$$

In the Equation (9), $D_{\text {eff }}$ is referred to the effective dispersion coefficient, which is superposed by molecular diffusion coefficient $D$ and Taylor dispersion coefficient $D_{\mathrm{T}}$ as [22],

$$
D_{\text {eff }}=D+D_{\mathrm{T}}=D\left[1+\frac{1}{210}\left(\frac{\bar{u} H}{D}\right)^{2}\right]
$$

Suppose the solution A with a biochemical factor concentration $\bar{\phi}_{\mathrm{A}}(t)$, and solvent B with no 
biochemical factor, the boundary conditions for the Equation (9) are as,

$$
\begin{array}{ll}
\text { B.C.1 : } & \left.\bar{\phi}(x, z, \mathrm{t})\right|_{z=0}=\bar{\phi}_{\mathrm{A}}(t), \quad \varepsilon W<x \leq W \\
\text { B.C.2 : } & \left.\bar{\phi}(x, z, \mathrm{t})\right|_{z=0}=0, \quad 0 \leq x \leq \varepsilon W \\
\text { B.C.3 : } & \partial \bar{\phi} /\left.\partial z\right|_{z \rightarrow \infty}=0, \quad \partial \bar{\phi} /\left.\partial x\right|_{x=0}=0, \quad \partial \bar{\phi} /\left.\partial x\right|_{x=W}=0
\end{array}
$$

The initial condition is:

$$
\left.\bar{\phi}(x, z>0, \mathrm{t})\right|_{t=0}=0
$$

\subsection{Microfluidic Device Fabrication and Experimental Setup}

A polydimethylsiloxane (PDMS)-glass Y-shaped microfluidic device is designed as shown in Figure 1a. The height $H$ of all the micro-channels is $80 \mu \mathrm{m}$, the width $W$ and the length $L$ of the mixing micro-channel $\mathrm{C}$ is $1000 \mu \mathrm{m}$ and $4 \mathrm{~cm}$, respectively. All the micro-channels were patterned in PDMS (Sylgard 184, Dow Corning, Midland, MI, USA) by replica molding. The mold was prepared by spin coating a thin layer of negative photoresist (SU-8, MicroChem, Westborough, MA, USA) onto a single side polishing silicon wafers and patterned with ultraviolet (UV) exposure. Next, the micro-channel layer was obtained by pouring PDMS with 10:1 $(w / w)$ base: crosslinker ratio onto the mold yielding a thickness of $3 \mathrm{~mm}$ roughly. After curing the elastomer for $2 \mathrm{~h}$ at $80^{\circ} \mathrm{C}$, the PDMS slab was peeled from the mold, punched and hermetically bonded to a coverslip by plasma oxidation.

As shown in Figure 2, the fabricated Y-shaped microfluidic chip (Figure 2a) connected with three syringe pumps (NE-1000, New Era Pump Systems, Inc., Farmingdale, NY, USA) for controlling the dynamic biochemical signal and the magnitude of WSS in mixing micro-channel $\mathrm{C}$ by regulating the flow rates from the three syringe pumps. More specifically, the inlet A was connected to two syringe pumps with a T-bend and silicone tubes (Figure $2 b$ ). The dynamic biochemical signal was generated by controlling the flow rates of the solution $\mathrm{A}$ and the solvent $\mathrm{A}$ from two syringe pumps, respectively. The inlet $\mathrm{B}$ was connected to the third syringe pump to generate time-varying laminar flow without biochemical factor. An inverted microscope (CKX41, Olympus Corporation, Tokyo, Japan) equipped with a CCD camera (DS126431, Canon Inc., Tokyo, Japan) was adopted to observe the biochemical signal and the intracellular calcium signal in vascular endothelial cells cultured on the bottom of the mixing channel $\mathrm{C}$ in real time (Figure 2c).

(a)

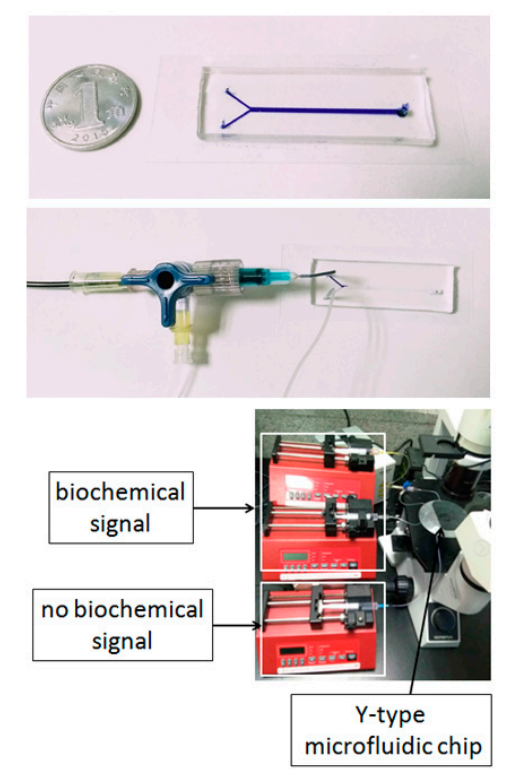

Figure 2. An actual Y-shaped microfluidic chip. (a) PDMS-glass microfluidic chip; (b) generator of dynamic biochemical signals; (c) the actual experimental setup. 


\subsection{Generation of Dynamic Biochemical Signals}

As shown in Figure 1c, the dynamic biochemical signal flowing into the inlet A was generated by controlling the flow rates of two syringes connected by a T-bend and two silicone tubes. The syringes were driven by two syringe pumps, respectively. Suppose that $Q_{\mathrm{A} 1}, Q_{\mathrm{A} 2}$ and $Q_{\mathrm{A}}$ were the flow rates of input solution $\mathrm{A}$, solvent $\mathrm{A}$ and output solution $\mathrm{A}$ respectively, and $\phi_{\mathrm{A} 1}$ and $\phi_{\mathrm{A}}$ were the concentrations of input solution $\mathrm{A}$ and output solution $\mathrm{A}$, the mass conservation law led to

$$
\begin{aligned}
& Q_{\mathrm{A} 1}+Q_{\mathrm{A} 2}=Q_{\mathrm{A}} \\
& Q_{\mathrm{A} 1} \phi_{\mathrm{A} 1}=Q_{\mathrm{A}} \phi_{\mathrm{A}}
\end{aligned}
$$

and then $Q_{\mathrm{A} 1}, Q_{\mathrm{A} 2}$ were expressed as

$$
Q_{\mathrm{A} 1}=\frac{Q_{\mathrm{A}} \phi_{\mathrm{A}}}{\phi_{\mathrm{A} 1}}
$$

and

$$
Q_{\mathrm{A} 2}=Q_{\mathrm{A}}\left(1-\frac{\phi_{\mathrm{A}}}{\phi_{\mathrm{A} 1}}\right)
$$

From Equations (14) and (15), a desired biochemical signal with the flow rate $Q_{\mathrm{A}}$ and the concentration $\phi_{\mathrm{A}}$ were implemented by controlling the syringe pumps by setting the flow rates $Q_{\mathrm{A} 1}$ and $Q_{\mathrm{A} 2}$ of syringes filled with the solution $\mathrm{A}$ and solvent $\mathrm{A}$, respectively. After solution $\mathrm{A}$ and solvent A was fully mixed, the biochemical signal was generated, and then delivered to the inlet A of the Y-shaped microfluidic chip through a short silicone tube (Figure $2 \mathrm{~b}$ ). In this delivering process, because the signal frequency is very low $(\sim 1 / 60 \mathrm{~Hz})$, the attenuation of the signal could be ignored before it reached the inlet $\mathrm{A}$ of the Y-shaped microfluidic device [23].

\subsection{Transport of Dynamic Biochemical Signals in the Mixing Micro-Channel C}

Before the dynamic biochemical signals input at the inlet A reached the mixing micro-channel C, they transported through a fully mixing microfluidic channel A which acts as a low-pass filter [24]. As the signal frequency in this study was as low as $1 / 60 \mathrm{~Hz}$, the effect of this fully mixing micro-channel A on the signal transportation was not considered. As the dynamic biochemical signals transported along the mixing micro-channel $C$, the spatiotemporal concentration profiles of biochemical signals were described by Equation (9) together with the boundary conditions (11). This subsection presents the numerical and experimental simulation studies about the transport of dynamic biochemical signals in the mixing micro-channel $\mathrm{C}$.

\subsubsection{Numerical Simulation}

For numerical simulation studies, Equation (9) was solved by a finite difference method. An Euler explicit discretization was used for the temporal derivation. The first-order and second-order central differences were adopted to approximate the first-order and second-order spatial derivation, respectively. Given the boundary conditions (11), i.e., the flow rates $Q_{\mathrm{A}}$ and $Q_{\mathrm{B}}(t)$ satisfying that $Q_{\mathrm{A}} / Q_{\mathrm{B}}(t)=(1-\varepsilon(t)) / \varepsilon(t)$, and the input signal $\bar{\phi}_{\mathrm{A}}(t)$, the spatiotemporal dynamic biochemical signal in the mixing micro-channel $C$ were numerically simulated using MATLAB (Version R2009b, The Math Works, Inc., Natick, MA, USA). All the simulation results were normalized to a constant reference value. In the numerical simulations, all the parameters for the Y-shaped microfluidic device and the solutions are listed in Table 1. 
Table 1. Default parameters used in the model.

\begin{tabular}{cc}
\hline Parameters & Values \\
\hline$L$ (z-direction) & $4 \mathrm{~cm}$ \\
$H$ (y-direction) & $80 \mu \mathrm{m}$ \\
$W$ (x-direction) & $1000 \mu \mathrm{m}$ \\
$\eta$ & $0.001 \mathrm{~Pa} \cdot \mathrm{s}$ \\
$D_{\text {fluorescent }}$ & $8.2 \times 10^{-10} \mathrm{~m}^{2} / \mathrm{s}$ \\
$D_{\text {ATP }}$ & $2.36 \times 10^{-10} \mathrm{~m}^{2} / \mathrm{s}$ \\
\hline
\end{tabular}

\subsubsection{Experimental Validation}

For actual experimental validation, the fluorescent solution (Rhodamine-6, Sigma-Aldrich, St. Louis, MO, USA) with time-dependent concentration was used to simulate the dynamic ATP signal. The fluorescent signal $\bar{\phi}_{\mathrm{A}}(t)$ was input through the inlet A of the Y-shaped microfluidic chip at a constant volumetric flow rate $\left(Q_{\mathrm{A}}=3.6 \mathrm{~mL} / \mathrm{h}\right)$. The volume flow rate $Q_{\mathrm{B}}(t)$ of solvent $B$ changes as a square wave with a period of $60 \mathrm{~s}$ between $Q_{\mathrm{A}}(3.6 \mathrm{~mL} / \mathrm{h})$ and $2 Q_{\mathrm{A}}(7.2 \mathrm{~mL} / \mathrm{h})$. The dynamic biochemical signal $\bar{\phi}_{\mathrm{A}}(t)$ from the inlet $\mathrm{A}$ synchronizes with the flow rate $Q_{\mathrm{B}}(t)$ from the inlet $\mathrm{B}$. The time-varying images for dynamic fluorescent signals at any positions in mixing channel $C$ could be observed and detected with the fluorescence microscope with the CCD camera (Figure 2c). The dynamic fluorescent intensities were then extracted from the images using MATLAB (The Math Works R2009b, Inc.). While the fluorescent intensities at each time point were calculated, the grey-values from the image background were subtracted. All the experimental results were normalized to a constant reference value.

\subsection{Cell Culture and Intracellular Calcium Dynamic Response}

HUVECs (derived from Dalian Medical University, Dalian, China) were cultured in Dulbecco's Modified Eagle's Medium (DMEM) (Invitrogen, Carlsbad, CA, USA) supplemented with $10 \%$ Fetal Bovine Serum (FBS) (Gibco, Thermo Fisher Scientific, Waltham, MA, USA) and were maintained at $37{ }^{\circ} \mathrm{C}$ with $5 \% \mathrm{CO}_{2}$ in culture flask. $0.25 \%$ Trypsin/EDTA (Gibco) was used to detach cells from plates and transfer them to the microfluidic chip as shown in Figure 2a. To ensure cell adhesion, the chip was subsequently filled with $100 \mathrm{mg} / \mathrm{mL}$ fibronectin (Sigma) and allowed to incubate at $37^{\circ} \mathrm{C}$ for two hours. Afterwards, the chip was flushed and refilled with DMEM supplemented with $10 \%$ FBS. HUVECs cells were then seeded in the mixing micro-channel $C$ from the outlet. The cells were then allowed to attach overnight. When HUVECs cells had been inoculated in the microfluidic chip for 4 days, the cytosolic calcium ions in cells were stained with $5 \mathrm{nM}$ Fluo-3 AM for $45 \mathrm{~min}$ in a culture medium at $37^{\circ} \mathrm{C}$. The cells were then rinsed with dye-free medium twice. The entire operation was performed with extreme caution to minimize the response of cells to early agitations. When microfluidic device was placed under microscope with a CCD camera, the syringe pumps would start up according to the designed program. The time-varying fluorescent images for the intracellular calcium response in HUVECs at the regions of interest were recorded in a sampling frequency (one frame per $4 \mathrm{~s}$ ) with the CCD for $3 \mathrm{~min}$ at room temperature. The calcium fluorescent intensities were then extracted from the dynamic images using the same method as described for dynamic fluorescent images in previous Section 2.4.2.

\section{Results}

\subsection{Spatiotemporal Profiles of Static and Dynamic Fluorescent Signals in the Mixing Micro-Channel C}

Figure 3 shows the spatiotemporal profiles of a static fluorescent signal transporting in a dynamic flow in the mixing micro-channel C. For this case, the input concentration $\bar{\phi}_{\mathrm{A}}(t)$ of solution $\mathrm{A}$ is a constant of $5 \mu \mathrm{mol} / \mathrm{mL}$, the volume flow rate $Q_{\mathrm{A}}$ is a constant of $3.6 \mathrm{~mL} / \mathrm{h}$ while the volume flow rate $Q_{\mathrm{B}}(t)$ is a dynamic signal as a square wave with a period of $60 \mathrm{~s}$ between 3.6 and $7.2 \mathrm{~mL} / \mathrm{h}$, and thus 
the WSS signal changes as a square wave with a period of $60 \mathrm{~s}$ between 1.875 and 2.813 Pa as well (Figure 3a). The spatial distribution of the fluorescent signal concentration at $t=15 \mathrm{~s}$ and at $t=45 \mathrm{~s}$ are exhibited in Figure 3b. It can be clearly seen from Figure $3 b$ that at any position along the length of the channel (z-direction), the fluorescent signal concentration keeps at $0 \mu \mathrm{mol} / \mathrm{mL}$ while $x$ is close to $0 \mathrm{~mm}$ and at a constant value $5 \mu \mathrm{mol} / \mathrm{mL}$ while $x$ is close to $1 \mathrm{~mm}$. The signal concentration will dramatically increase from 0 to $5 \mu \mathrm{mol} / \mathrm{mL}$ along $x$-direction at the region around the interface between two streams from the inlet $\mathrm{A}$ and $\mathrm{B}$. Besides, because the volume flow rate $Q_{\mathrm{B}}(t)$ dynamically changes as a square-wave like signal, this interface also dynamically changes its position along $x$-direction. These numerical simulation results can be validated by experimental results as shown in Figure 3c.

(a)

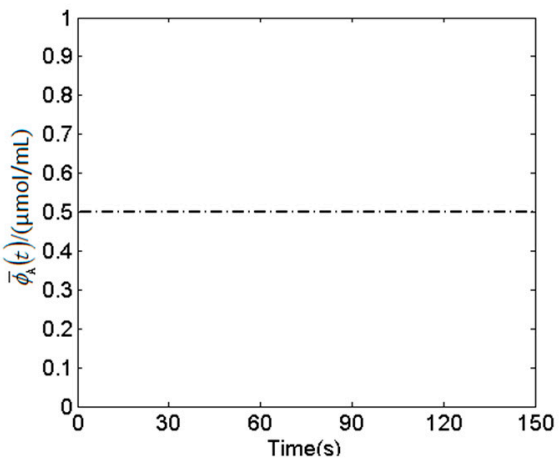

(b)

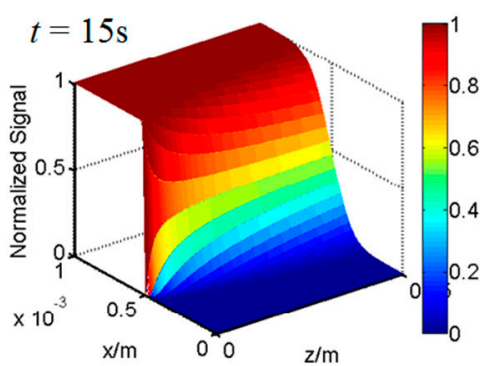

$15 s$
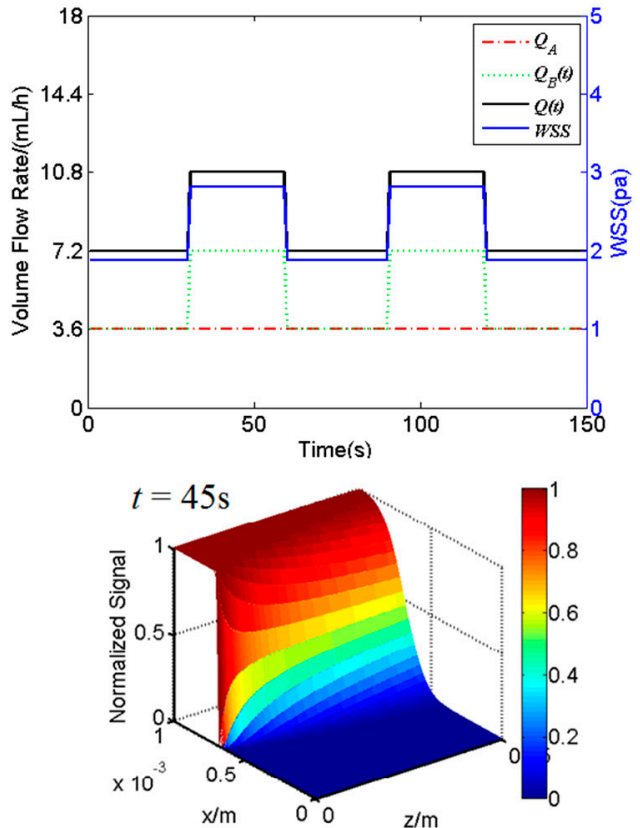

$45 s$

(c)
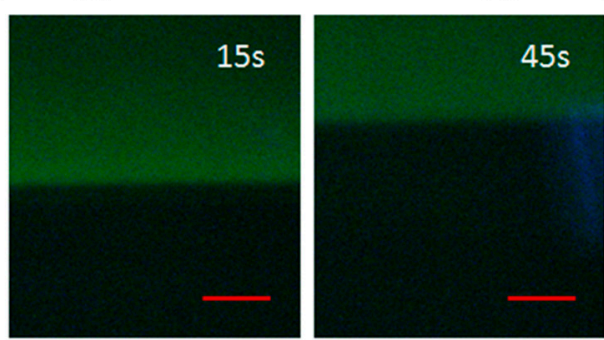

Figure 3. An input static fluorescent signal and its spatiotemporal concentration profile in a dynamic flow in the mixing micro-channel C. (a) The static fluorescent signal $\bar{\phi}_{\mathrm{A}}(t)$, the volume flow rates $Q_{\mathrm{A}}$, $Q_{\mathrm{B}}(t), Q(t)$ and wall shear stress (WSS); (b) numerically simulated concentration profile at $t=15 \mathrm{~s}$ and $t=45 \mathrm{~s}$ in $x-z$ plane, respectively; (c) experimental concentration profiles at $t=15 \mathrm{~s}$ and $t=45 \mathrm{~s}$ in $x-z$ plane, respectively. Scale bar is $100 \mu \mathrm{m}$.

The spatiotemporal concentration profiles of a square-wave-like fluorescent signal transporting in a steady flow in the mixing micro-channel $\mathrm{C}$ are illustrated in Figure 4 . Under the steady flow, the concentration $\bar{\phi}_{\mathrm{A}}(t)$ of solution $\mathrm{A}$ is a dynamic square wave with a period of $60 \mathrm{~s}$ between $5 \mu \mathrm{mol} / \mathrm{mL}$ and $0 \mu \mathrm{mol} / \mathrm{mL}$ (Figure 3a). As shown in Figure $4 \mathrm{a}$, for the steady flow, the volume flow rates $Q_{\mathrm{A}}$ and $Q_{\mathrm{B}}(t)$ are the same as $3.6 \mathrm{~mL} / \mathrm{h}$ and thus the WSS is constant at $1.875 \mathrm{~Pa}$. Figure $4 \mathrm{~b}$ shows the concentration profile at $z=2 \mathrm{~cm}$ in $x$ - $t$ plane under steady flow. It is obvious in Figure $4 \mathrm{~b}$ that the dynamic fluorescent signal keeps square-wave-like at the region that $x$ is close to $1 \mathrm{~mm}$, but the amplitude of the signal at the region around the interface between two streams from the inlet $\mathrm{A}$ and B decreases due to transverse molecular diffusion. Furthermore, the dynamic fluorescent signal 
has no significant amplitude attenuation and phase delay while it is transporting along the mixing micro-channel (data not shown). In addition, this interface keeps its position along $x$-direction under steady flow. All these numerical simulation results can also be reproduced by experimental results as shown in Figure 4c.

(a)

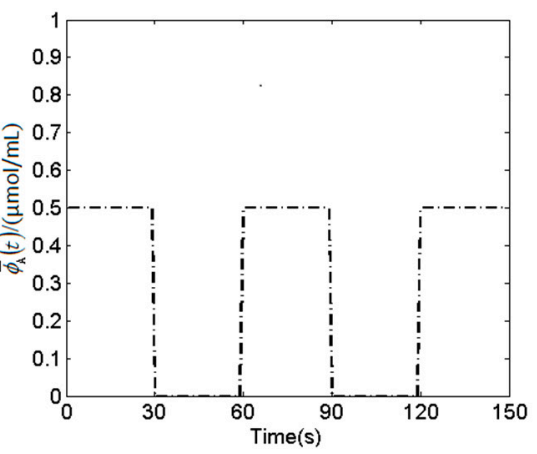

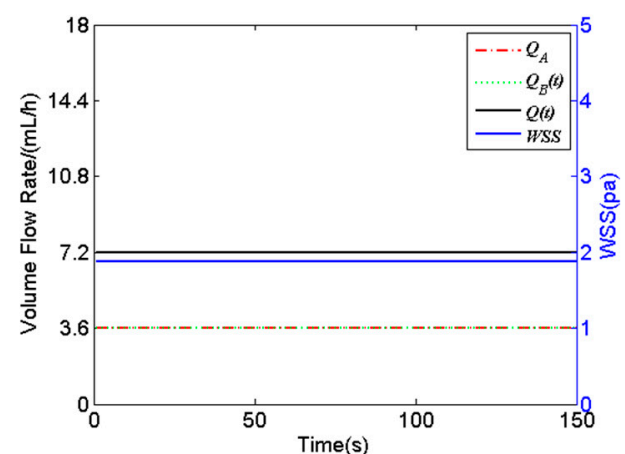

(b)

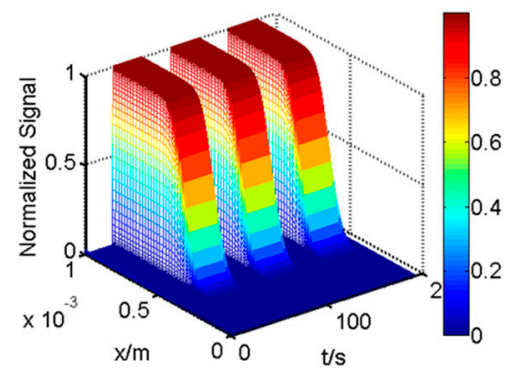

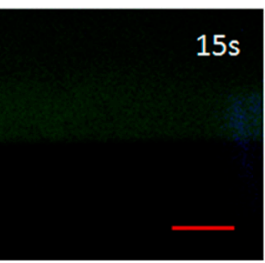
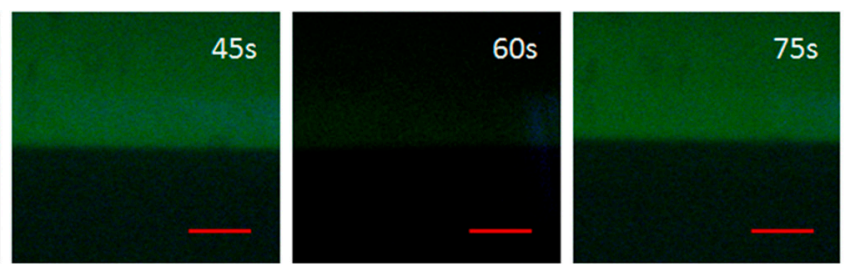

Figure 4. An input dynamic fluorescent signal and its spatiotemporal concentration profile in steady flow in the mixing micro-channel C. (a) The dynamic fluorescent signal $\bar{\phi}_{\mathrm{A}}(t)$ and the volume flow rates $Q_{\mathrm{A}}, Q_{\mathrm{B}}(t), \mathrm{Q}(t)$ and WSS; (b) numerically simulated spatiotemporal concentration profiles at $z=2 \mathrm{~cm}$ in $x$-t plane under steady flow; (c) experimental spatiotemporal concentration profiles at the region around $x=2 \mathrm{~cm}$ in $x$-z plane under steady flow. Scale bar is $100 \mu \mathrm{m}$.

\subsection{Combination of WSS and Fluorescent Signals at the Central Regime Along the Mixing Micro-Channel C}

It can be clearly observed from Figures 3 and 4 that at any $z$ position along the mixing micro-channel $C$, there exist different regimes along $x$-direction where the transporting WSS and/or fluorescent signals are different. Figure 5 shows the comparison between simulation results and experimental data of combined WSS and fluorescent signals at three $(x=W / 8, W 5 / 8$ and $W 7 / 8$ in Figure $5 a)$ or two $(x=W / 8$ and $W 7 / 8$ in Figure $5 b)$ different locations, at the central regime $(z=2 \mathrm{~cm})$ in the mixing micro-channel C. It can be easily seen form Figure 5 that different combinations of WSS and fluorescent signals are produced in the mixing micro-channel. More specifically, Figure 5a exhibits the dynamic WSS alone at $x=W / 8$, the combination of dynamic WSS and dynamic fluorescent signal at $x=W 5 / 8$, and the combination of dynamic WSS and static fluorescent signal at $x=W 7 / 8$; Figure $5 \mathrm{~b}$ illustrates the static WSS alone at $x=W / 8$ and the combination of static WSS and dynamic fluorescent signal at $x=W 7 / 8$, respectively. All the fluorescent signals in Figures 4 and 5 were replaced by ATP signals instead in the HUVECs calcium dynamic response experiments as shown in Figures 6 and 7. 
(a)

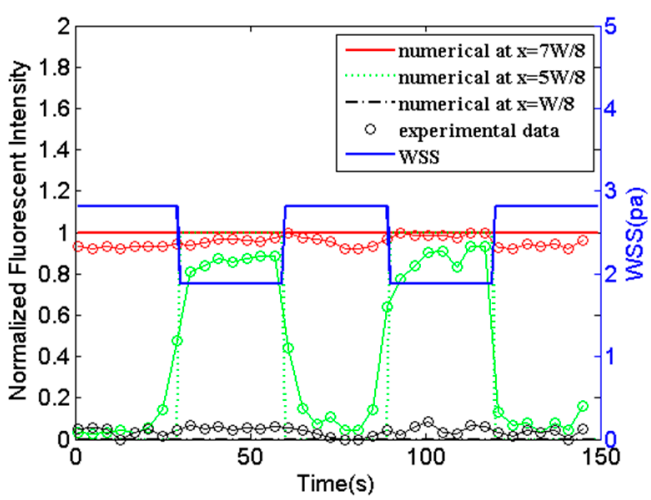

(b)

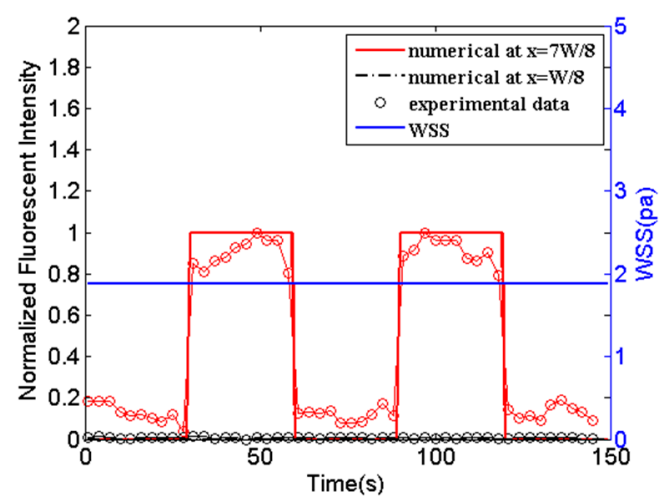

Figure 5. Comparison between simulation results and experimental data of combined WSS and fluorescent signals at different locations at $z=2 \mathrm{~cm}$ in the mixing micro-channel C. (a) Static fluorescent signal under dynamic flow; (b) dynamic fluorescent signal under steady flow. All the signals are normalized to a constant reference value.

\subsection{Intracellular $\mathrm{Ca}^{2+}$ Dynamics in Huvecs in Response to Combined Effects of WSS and ATP Signals}

Figure 6a shows the images of the HUVECs were cultured on the bottom of mixing micro-channel for 4 days. After incubated with $5 \mathrm{nM}$ Fluo3-AM for $45 \mathrm{~min}$, the dynamic responses of the intracellular $\mathrm{Ca}^{2+}$ concentration induced by static or dynamic WSS alone, as well as different combinations of WSS and ATP signals were carefully measured. As a specific case, Figure $6 \mathrm{~b}$ exhibits the intracellular $\mathrm{Ca}^{2+}$ intensity at $3 \mathrm{~s}, 24 \mathrm{~s}, 69 \mathrm{~s}$, and $111 \mathrm{~s}$, respectively, in the HUVECs at $z=2 \mathrm{~cm}$ in the mixing micro-channel $C$ under the stimulation of a combination of static ATP signal $(1 \mu \mathrm{mol} / \mathrm{L})$ and dynamic WSS (with the period of $60 \mathrm{~s}$ ).

Different combined effects of WSS and ATP signals on the intracellular $\mathrm{Ca}^{2+}$ dynamics in HUVECs are shown in Figure 7. Figure 7a demonstrates that intracellular $\mathrm{Ca}^{2+}$ response dynamics under the stimulation of static WSS signal alone at $x=W / 8$ under the condition that volume flow rate is constant. It can be seen from Figure 7a that after motivated by this static WSS signal alone, as increases in time, the intracellular $\mathrm{Ca}^{2+}$ concentration increases to a maximum and then deceases to the original value. Only single peak $\mathrm{Ca}^{2+}$ response is observed for this case. However, once this static WSS signal co-works with a dynamic ATP signal, a second dynamic response of intracellular $\mathrm{Ca}^{2+}$ concentration with the same frequency as that of ATP signal can be observed at $x=W 7 / 8$ of the micro-channel C as shown in Figure $7 \mathrm{~b}$. Similar dynamic response of the intracellular $\mathrm{Ca}^{2+}$ concentration can also be found in HUVECs activated by a dynamic WSS alone (Figure 7c) and a combination of static ATP signal and dynamic WSS (Figure 7d) at the religion $x=W / 8$ and $x=W 7 / 8$ respectively.

It is easy to observe that the intracellular $\mathrm{Ca}^{2+}$ dynamic responses in HUVECs in exposure to combined effect of WSS and ATP synchronizes with the stimulating dynamic WSS or dynamic ATP signal, however, dynamic WSS or dynamic ATP signal alone cannot induce much more transient $\mathrm{Ca}^{2+}$ responses. These interesting results demonstrate that the synergistic effect of WSS and ATP signals might play a critical role in the HUVEC signal transduction. Further investigations will be required to understand this phenomenon and the underlying molecular mechanism. 
(a)

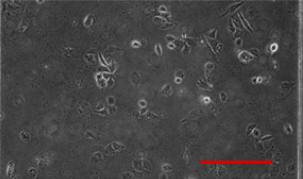

1d

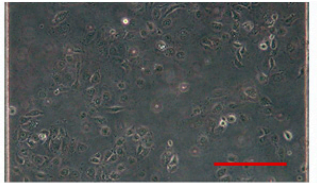

$2 d$

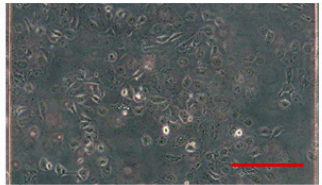

$4 d$
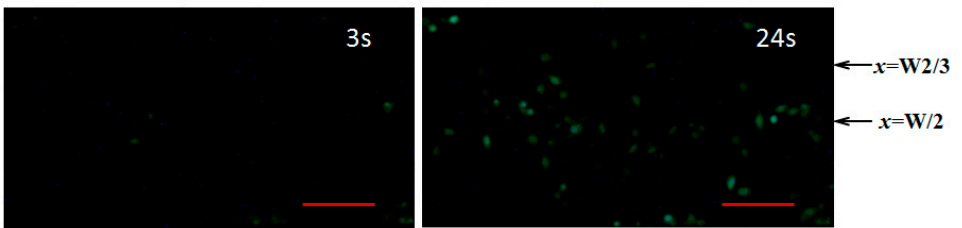

(b)
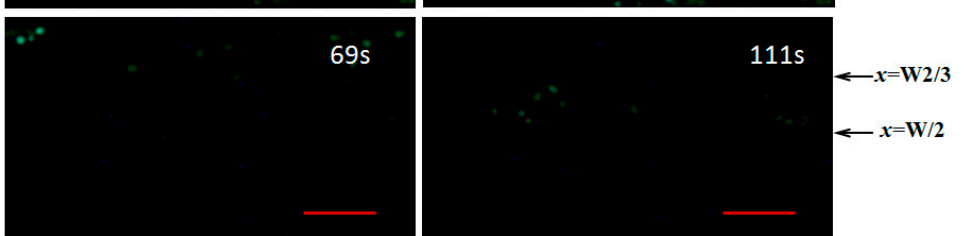

Figure 6. The intracellular $\mathrm{Ca}^{2+}$ response in the human umbilical vein endothelial cells (HUVECs) culture on the bottom of the mixing micro-channel $C$ at $z=2 \mathrm{~cm}$. (a) HUVECs cultured for $1 \mathrm{~d}, 2 \mathrm{~d}$ and $4 \mathrm{~d}$, respectively; (b) the intracellular $\mathrm{Ca}^{2+}$ intensity at $t=3 \mathrm{~s}, 24 \mathrm{~s}, 69 \mathrm{~s}$, and $111 \mathrm{~s}$, respectively. Scale bar is $100 \mu \mathrm{m}$.

(a)

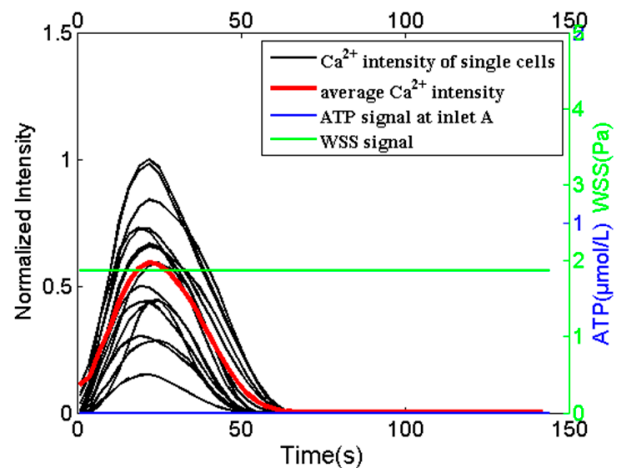

(c)

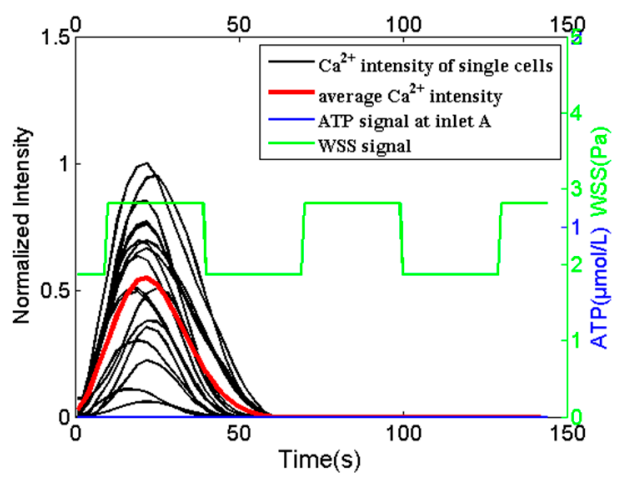

(b)

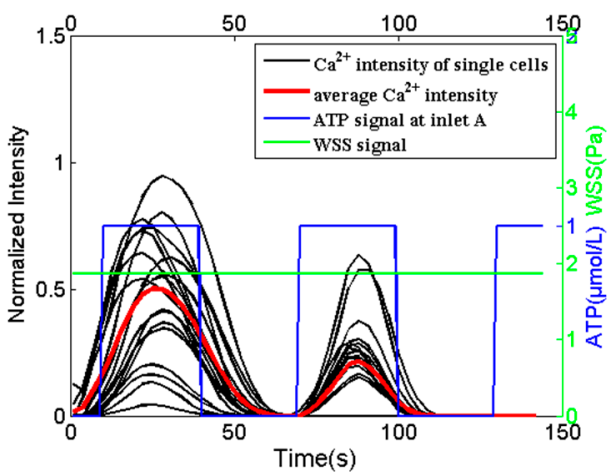

(d)

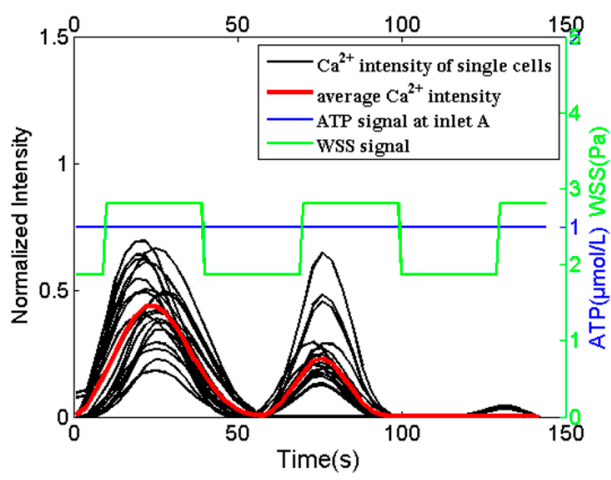

Figure 7. The intracellular $\mathrm{Ca}^{2+}$ dynamics in HUVECs in response to WSS signal alone and combined WSS and adenosine triphosphate (ATP) signals. (a) static WSS signal alone and (b) static WSS signal with dynamic ATP signal at the religion of $x=W / 8$ or $x=W 7 / 8$ in mixing micro-channel $C$ under the condition that volume flow rate is constant, respectively; (c) dynamic WSS signal alone and (d) dynamic WSS signal with static ATP signal at the religion of $x=W / 8$ or $x=W 7 / 8$ in mixing micro-channel C under the condition that volume flow rate is dynamic changing, respectively. Experimental data in $(\mathbf{a}, \mathbf{b})$ are measured from one group of HUVECs while those in $(\mathbf{c}, \mathbf{d})$ are measure from another group of HUVECs. The average $\mathrm{Ca}^{2+}$ intensity is the average value of 20 single cells. All the data are normalized to a constant reference value. 


\section{Discussion}

A Y-shaped microfluidic device, which provides different combinations of WSS and ATP signals in one single micro-channel, is proposed based upon the principles of fluid mechanics and mass transfer. This Y-shaped microfluidic device has an inlet A to input static or dynamic ATP signal, an inlet B to input static or dynamic laminar flow, and a mixing micro-channel for cell culture. The combinations of WSS and ATP signals are generated by controlling the volume flow rates of three programmable syringe pumps. To the best of our knowledge, such a Y-shaped microfluidic device is firstly used to study the combined effects of WSS and ATP signals, especially the impact of dynamic ATP signals, on intracellular $\mathrm{Ca}^{2+}$ dynamic in VECs although this type of Y-shaped microfluidic chip has been commonly adopted in biochemical mixing [25,26], cell sorting [27-29] and rapid biochemical switching for analysis at the cellular level $[16,17]$.

It is of importance to understand the transport characteristics of the dynamic biochemical signals transporting in fluid flows in the micro-channels of the Y-shaped microfluidic device in order to precisely load biochemical signals on the desired cells cultured on the bottom of the mixing micro-channel as these microfluidic channels act as low-pass filters [22]. The WSS signals and the spatiotemporal concentration profiles of biochemical signals in the mixing micro-channel are carefully analyzed by numerically solving the equations governing the dynamic laminar flow and time-dependent Taylor-Aris dispersion (see Figures 3-5). Numerical simulation results demonstrate that the WSS signals on the bottom of the mixing micro-channel are the same. The biochemical signals with low frequency (e.g., 1/60 Hz) have very little amplitude attenuation and phase delay while transporting in steady flow in the silicone tube or the micro-channels (data not shown), which is consistent with the conclusion in the literature [22-24]. Thus, the concentration profiles of a static or dynamic biochemical signal at any locations along the length of the mixing micro-channel (z-direction) are almost the same, which ensures that the cultured cells around the central regime $(z \approx 2 \mathrm{~cm})$ along $z$-direction are in exposure to very similar and stable biochemical signals (see Figures $3 c$ and $4 c$ ). However, at any $z$ positions and along $x$-direction, the biochemical signal switches dramatically from zero to a stationary signal around the interface between two streams from the inlet A and B, resulting in two (Figure 5b) or three (Figure 5a) regimes with WSS signal alone or different combinations of WSS and biochemical signals as shown in Figure 5. All these numerical simulation results can be validated by using fluorescent simulation experiments, demonstrating the Y-shaped microfluidic device can provide the culture cells on the bottom of the mixing channel with desired WSS and ATP signals as well as their combinations.

Using the proposed Y-shaped microfluidic device, the different combined effects of WSS and ATP signal on the intracellular $\mathrm{Ca}^{2+}$ dynamics in HUVECs culture on the bottom of the mixing micro-channel are measured. The experimental results suggest that the intracellular $\mathrm{Ca}^{2+}$ dynamic responses in HUVECs in exposure to combined effect of WSS and ATP synchronizes with the stimulating dynamic WSS or dynamic ATP signals, however, dynamic WSS or dynamic ATP signal alone cannot induce much more transient $\mathrm{Ca}^{2+}$ responses. These interesting results demonstrate that the synergistic effect of WSS and ATP signals, but not WSS or ATP signal alone, might play a critical role in the HUVEC signal transduction. A potential mechanism for this synergistic effect is that the inflowing ATP together with endogenously released ATP from HUVECs by wall shear stress activated P2X/P2Y signaling pathways, which in turn induced calcium release from calcium stores [5-7,30]; meanwhile, the mechano-sensitive channels on cell membrane directly activated by wall shear stress, leading to extracellular calcium influx across the cell membrane [11,31]; these two signaling pathways would interplay with each other. Further studies are necessary to confirm this novel phenomenon and figure out the underlying molecular mechanism. 


\section{Conclusions}

A Y-shaped microfluidic device is proposed to investigate the combined effects of WSS and ATP signals on the intracellular $\mathrm{Ca}^{2+}$ dynamics in VECs. The Y-shaped microfluidic devices can provide the cultured cells on the bottom of its mixing micro-channel with stimuli of WSS signal alone and different combinations of WSS and ATP signals in one single micro-channel, which are validated by both numerical and experimental simulation studies. Cellular $\mathrm{Ca}^{2+}$ dynamic response experiments also verify the feasibility of application of the device. Preliminary experimental results of intracellular $\mathrm{Ca}^{2+}$ dynamics show that a combination of WSS and ATP signals rather than a WSS signal alone might play a more important role in $\mathrm{VEC} \mathrm{Ca}^{2+}$ signal transduction induced by blood flow.

Acknowledgments: The authors would like to appreciate Wenyu Liu from Dalian University of Technology for English Editing. The project is, in part, supported by the National Natural Science Foundation of China (Nos. 11172060, 31370948, 11672065), State Natural Science Foundation of Liaoning (2015020303), and Science \& Technology Foundation of Dalian (2015E12SF167).

Author Contributions: Z.-Z.C. and K.-R.Q. conceived and designed the experiments; Z.-Z.C. performed the experiments; Z.-Z.C., Z.-M.G. and D.-P.Z. analyzed the data; Y.L. and B.L. gave scientific support and conceptual advices; Z.-Z.C. and K.-R.Q. wrote the paper. All authors discussed the results and implications and commented on the manuscript.

Conflicts of Interest: The authors declare no conflict of interest.

\section{Abbreviations}

$\begin{array}{ll}\text { WSS } & \text { Wall shear stress } \\ \text { ATP } & \text { Adenosine triphosphate } \\ \text { VECs } & \text { Vascular endothelial cells } \\ D & \text { Diffusivity of solute }\left(\mathrm{m}^{2} / \mathrm{s}\right) \\ D_{\text {eff }} & \text { Effective diffusivity coefficient of solute }\left(\mathrm{m}^{2} / \mathrm{s}\right) \\ H & \text { Height of the mixing micro-channel }(\mathrm{m}) \\ p & \text { Pressure }(\mathrm{Pa}) \\ Q & \text { Total flow rate in the mixing micro-channel C }(\mathrm{mL} / \mathrm{h}) \\ Q_{\mathrm{A}} & \text { Flow rate at inlet A }(\mathrm{mL} / \mathrm{h}) \\ Q_{\mathrm{B}}(t) & \text { Flow rate at inlet B }(\mathrm{mL} / \mathrm{h}) \\ u & \text { Velocity of fluid in } z \text { direction }(\mathrm{m} / \mathrm{s}) \\ W & \text { Width of the mixing micro-channel }(\mathrm{m}) \\ W_{1} & \text { Width of solution A in the mixing micro-channel }(\mathrm{m}) \\ W_{2} & \text { Width of solvent B in the mixing micro-channel }(\mathrm{m}) \\ \varepsilon(t) & \text { Ratio of } W_{2} \text { to } W \\ \eta & \text { Viscosity of fluid }(\text { Pa } \cdot \mathrm{s}) \\ \bar{\phi}_{\mathrm{A}}(t) & \text { Concentration of solution in the micro-channel }(\mu \mathrm{mol} / \mathrm{mL}) \\ \bar{\phi}(t) & \text { Height-wise averaging concentration of solution }(\mu \mathrm{mol} / \mathrm{mL}) \\ \bar{\phi}_{\mathrm{A}}(t) & \text { Height-wise averaging concentration of solution at inlet A }(\mu \mathrm{mol} / \mathrm{mL}) \\ \tau_{\mathrm{w}}(t) & \text { Wall shear stress }(\text { Pa) }\end{array}$

\section{References}

1. Ando, J.; Ohtsuka, A.; Korenaga, R.; Kamiya, A. Effect of extracellular ATP level on flow-induced Ca ${ }^{++}$ response in cultured vascular endothelial cells. Biochem. Biophys. Res. Commun. 1991, 179, 1192-1199. [CrossRef]

2. Ando, J.; Kamiya, A. Cytoplasmic calcium response to fluid shear stress in cultured vascular endothelial cells. In Vitro Cell. Dev. Biol. 1988, 24, 871-877. [CrossRef] [PubMed]

3. Dull, R.O.; Davies, P.F. Flow modulation of agonist (ATP)-response $\left(\mathrm{Ca}^{2+}\right)$ coupling in vascular endothelial cells. Am. J. Physiol. 1991, 261, 149-154.

4. Mo, M.; Eskin, S.G.; Schilling, W.P. Flow-induced changes in $\mathrm{Ca}^{2+}$ signaling of vascular endothelial cells: Effect of shear stress and ATP. Am. J. Physiol. 1991, 260, 1698-1707.

5. Yamamoto, K.; Korenaga, R.; Kamiya, A.; Ando, J. Fluid shear stress activates $\mathrm{Ca}^{2+}$ influx into human endothelial cells via P2X4 purinoceptors. Circ. Res. 2000, 87, 385-391. [CrossRef] [PubMed]

6. Yamamoto, K.; Korenaga, R.; Kamiya, A.; Qi, Z.; Sokabe, M.; Ando, J. P2X4 receptors mediate ATP-induced calcium influx in human vascular endothelial cells. Am. J. Physiol. Heart Circ. 2000, 279, 285-292. 
7. Yamamoto, K.; Sokabe, T.; Ohura, N.; Nakatsuka, H.; Kamiya, A.; Ando, J. Endogenously released ATP mediates shear stress-induced $\mathrm{Ca}^{2+}$ influx into pulmonary artery endothelial cells. Am. J. Physiol. Heart Circ. 2003, 285, 793-803. [CrossRef] [PubMed]

8. Shen, J.; Luscinskas, F.W.; Gimbrone, M.A.; Dewey, C.F. Fluid flow modulates vascular endothelial cytosolic calcium responses to adenine nucleotides. Microcirculation 1994, 1, 67-78. [CrossRef] [PubMed]

9. James, N.L.; Harrison, D.G.; Nerem, R.M. Effects of shear on endothelial cell calcium in the presence and absence of ATP. FASEB J. 1995, 9, 968-973. [PubMed]

10. Helmlinger, G.; Berk, B.C.; Nerem, R.M. Pulsatile and steady flow-induced calcium oscillations in single cultured endothelial cells. J. Vasc. Res. 1996, 33, 360-369. [CrossRef] [PubMed]

11. Li, L.F.; Xiang, C.; Qin, K.R. Modeling of TRPV4-C1-mediated calcium signaling in vascular endothelial cells induced by fluid shear stress and ATP. Biomech. Model. Mechanbiol. 2015, 14, 979-993. [CrossRef] [PubMed]

12. Davies, P.F. Flow-mediated endothelial mechanotransduction. Physiol. Rev. 1995, 75, 519-560. [PubMed]

13. Berridge, M.J.; Lipp, P.; Bootman, M.D. The versatility and universality of calcium signaling. Nat. Rev. Mol. Cell Biol. 2000, 1, 11-21. [CrossRef] [PubMed]

14. Clapham, D.E. Calcium signaling. Cell 1995, 80, 259-268. [CrossRef]

15. Bibhas, R.; Tamal, D.; Debasish, M.; Maiti, T.K.; Chakraborty, S. Oscillatory shear stress induced calcium flickers in osteoblast cells. Integr. Biol. 2014, 6, 289-299.

16. Yamada, A.; Katanosaka, Y.; Mohri, S.; Naruse, K. A rapid microfluidic switching system for analysis at the single cellular level. IEEE Trans. Nanobiosci. 2009, 8, 306-311. [CrossRef] [PubMed]

17. Kuczenski, B.; Ruder, W.C.; Messner, W.C.; Leduc, P.R. Probing cellular dynamics with a chemical signal generator. PLoS ONE 2009, 4, e4847. [CrossRef] [PubMed]

18. Zhang, X.; Grimley, A.; Bertram, R.; Roper, M.G. Microfluidic system for generation of sinusoidal glucose waveforms for entrainment of islets of Langerhans. Anal. Chem. 2010, 82, 6704-6711. [CrossRef] [PubMed]

19. Kim, Y.T.; Joshi, S.D.; Messner, W.C.; LeDuc, P.R.; Davidson, L.A. Detection of dynamic spatiotemporal response to periodic chemical stimulation in a Xenopus Embryonic tissue. PLoS ONE 2011, 6, e14624. [CrossRef] [PubMed]

20. Shin, H.; Mahto, S.K.; Kim, J.H.; Rhee, S.W. Exposure of BALB/3T3 fibroblast cells to temporal concentration profile of toxicant inside microfluidic device. Biochip J. 2011, 5, 214-219. [CrossRef]

21. Wang, Y.X.; Xiang, C.; Liu, B.; Zhu, Y.; Luan, Y.; Liu, S.T.; Qin, K.R. A multi-component parallel-plate flow chamber system for studying the effect of exercise-induced wall shear stress on endothelial cells. Biomed. Eng. 2016. submitted for publication.

22. Li, Y.J.; Li, Y.Z.; Cao, T.; Qin, K.R. Transport of dynamic biochemical signals in steady flow in a shallow Y-shaped microfluidic channel: Effect of transverse diffusion and longitudinal dispersion. J. Biomech. Eng. 2013, 135, 121011-121019. [CrossRef] [PubMed]

23. Qin, K.R.; Xiang, C.; Ge, S.S. Generation of dynamic biochemical signals with a tube mixer: Effect of dispersion in an oscillatory flow. Heat Mass Transf. 2010, 46, 675-686. [CrossRef]

24. Xie, Y.; Wang, Y.; Mastrangelo, C.H. Fourier microfluidics. Lab Chip 2008, 8, 779-785. [CrossRef] [PubMed]

25. Capretto, L.; Cheng, W.; Hill, M.; Zhang, X. Micromixing within microfluidic devices. Top. Curr. Chem. 2011, 304, 27-68. [PubMed]

26. Nguyen, N.T.; Wu, Z. Micromixers-A review. J. Micromech. Microeng. 2005, 15, 1-16. [CrossRef]

27. Furdui, V.I.; Harrison, D.J. Immunomagnetic T cell capture from blood for PCR analysis using microfluidic systems. Lab Chip 2004, 4, 614-618. [CrossRef] [PubMed]

28. Mazutis, L.; Gilbert, J.; Ung, W.L.; Weitz, D.A.; Griffiths, A.D.; Heyman, J.A. Single-cell analysis and sorting using droplet-based microfluidics. Nat. Protoc. 2013, 8, 870-891. [CrossRef] [PubMed]

29. Yamada, M.; Nakashima, M.; Seki, M. Pinched flow fractionation: Continuous size separation of particles utilizing a laminar flow profile in a pinched microchannel. Anal. Chem. 2004, 76, 5465-5471. [CrossRef] [PubMed] 
30. Malek, A.M.; Zhang, J.; Jiang, J.; Alper, S.L.; Izumo, S. Endothelin-1 gene suppression by shear stress: Pharmacological evaluation of the role of tyrosine kinase, intracellular calcium, cytoskeleton, and mechanosensitive channels. J. Mol. Cell. Cardiol. 1999, 31, 387-399. [CrossRef] [PubMed]

31. Kanai, A.J.; Strauss, H.C.; Truskey, G.A.; Crews, A.L.; Grunfeld, S.; Malinski, T. Shear stress induces ATP-independent transient nitric oxide release from vascular endothelial cells, measured directly with a porphyrinicmicrosensor. Circ. Res. 1995, 77, 284-293. [CrossRef] [PubMed]

(C) 2016 by the authors; licensee MDPI, Basel, Switzerland. This article is an open access article distributed under the terms and conditions of the Creative Commons Attribution (CC-BY) license (http:/ / creativecommons.org/licenses/by/4.0/). 